\title{
Analgesia caudal continua guiada por ultrasonido en una paciente de 4 años
}

\author{
A. Beltrán Francoํㅡㄹ M. M. Pelaez Hernández¹, C. E. Restrepo-Garcés², C. M. Gómez Bermúdez ${ }^{3}$ \\ y G. A. Molina ${ }^{4}$
}

${ }^{\text {I} P r o g r a m a ~ d e ~ A n e s t e s i o l o g i ́ a ~ y ~ R e a n i m a c i o ́ n . ~ U n i v e r s i d a d ~ P o n t i f i c i a ~ B o l i v a r i a n a . ~}{ }^{2}$ Clínica de Alivio del Dolor. Departamento de Cirugía. Departamento de Imágenes Diagnósticas. Hospital Pablo Tobón Uribe. Unidad de Alivio del Dolor. Departamento de Anestesiología. Clínica Las Américas. Instituto de Cancerología. ${ }^{3}$ Clínica de Alivio del Dolor. Departamento de Cirugía. Departamento de Imágenes Diagnósticas. Hospital Pablo Tobón Uribe. Unidad de Alivio del Dolor. Departamento de Anestesiología. Clínica Las Américas. ${ }^{4}$ Ortopedia Oncológica. Departamento de Ortopedia. Hospital Pablo Tobon Uribe. Ortopedia Oncológica. Clínica Las Américas e Instituto de Cancerología. Medellín, Colombia.

Beltrán Franco A, Pelaez Hernández MM, RestrepoGarcés CE, Gómez Bermúdez CM, Molina GA. Analgesia caudal continua guiada por ultrasonido en una paciente de 4 años. Rev Soc Esp Dolor 2013; 20(1): 34-39.

\begin{abstract}
Caudal epidural is the most popular regional analgesia and anesthesia technique in pediatrics. The use of ultrasound (US) guidance in this procedure, is not yet the standard, but could reduce the risks related with the traditional approach and offer some advantages. We described a case of a 4-years-old patient undergoing a resection of a rabdomyosarcome on the left thigh plus inguinal metastatic nodes and implantation of brachitherapy catheters, in whom a continous caudal epidural catheter was placed under US guidance. After general anesthesia induction, a scout scanning identified the anatomy and afterwards, using strict aseptic techniques a caudal catheter was indwelling under the US guidance on real time and using the Doppler mode confirm the position of it inside the caudal epidural space with a local anesthetic bolus. There was an optimal pain control after surgery. The use of US as a guidance tool for caudal epidural catheter placement is an excellent alternative to the classic anatomical landmarks and give some advantages compare with those blind techniques.
\end{abstract}

Financiación: recursos propios. Conflicto de intereses: ninguno.
Key words: Pediatrics. Children. Caudal anesthesia. Epidural. Regional anesthesia. Ultrasonography. Continuos infusion.

\section{RESUMEN}

La epidural caudal es la técnica más popular en anestesia y analgesia regional pediátrica. El empleo de una guía ecográfica en este procedimiento, aunque aún no es un estándar, podría disminuir los riesgos inherentes a la técnica tradicional y ofrecer algunas ventajas. Nosotros describimos el caso de una niña de 4 años sometida a una resección de un rabdomiosarcoma en muslo izquierdo con metástasis ganglionar inguinal e implantación de catéteres para braquiterapia; a quien se le colocó un catéter caudal para analgesia postoperatoria continua, usando la ultrasonografía (US) como método para guiar la colocación de dicho catéter. Después de inducir anestesia general, se realizó un escaneo ecográfico previo de la zona sacra identificando la anatomía, posteriormente después de implementar las medidas antisépticas y asépticas se colocó un catéter caudal guiado por US en tiempo real y con modo Doppler color se confirmó la posición en el espacio epidural caudal al inyectar una dosis en bolo de mezcla anestésica. Se presentó un adecuado control del dolor postoperatorio. El uso de US es una excelente alternativa a las técnicas clásicas fundamentadas en anatomía para la inserción de catéteres epidurales continuos en pediatría y permite ciertas ventajas que las técnicas a ciegas no pueden brindar.

Palabras clave: Pediátrico. Niños. Anestesia caudal. Epidural. Anestesia regional. Ultrasonido. Infusión continua. 


\section{INTRODUCCIÓN}

El bloqueo caudal es la técnica más popular en anestesia y analgesia regional pediátrica (1) por ser una técnica fácil, rápida y efectiva; que ofrece óptima calidad anestésica/analgésica en procedimientos mayores y menores. En Latinoamérica, la técnica es fundamentada en referencias anatómicas, la cual es usada ampliamente; siendo la dosis única por aguja, la más utilizada. En la literatura latinoamericana, solo una revisión reciente resalta las bondades del ultrasonido (US) en el bloqueo caudal con dosis única no solo en niños sino en adultos (2). Por el contrario el uso de técnicas continuas con catéteres es menos popular y hasta ahora no existen reportes latinoamericanos de catéter continuo guiado por US. Esta situación no difiere mucho de otras latitudes más desarrolladas, donde según una encuesta reciente realizada entre anestesiólogos pediátricos del Reino Unido solo el 7,3\% de ellos usan la US en el abordaje caudal (3). La técnica por US para guiar la colocación de un catéter caudal en niños ha sido revisada ampliamente por Ban C. H. Tsui y cols., recientemente (4). Es llamativo que a pesar del uso masivo de las técnicas guiadas por US en anestesia y analgesia regional periférica, el papel del US en técnicas neuroaxiales pediátricas es mínimo, desaprovechando sus bondades, particularmente en esta población (5). El empleo de una guía ecográfica en este procedimiento, aunque aún no puede ser considerado la aproximación estándar, podría disminuir los riesgos inherentes a la técnica tradicional y ofrecer ventajas, como disminuir el contacto óseo y además permite evaluar el adecuado posicionamiento de la aguja y del catéter, entre otros (6). Nosotros describimos el caso de una niña de 4 años sometida a resección de rabdomiosarcoma en muslo izquierdo con metástasis ganglionar inguinal en la que se empleó analgesia caudal continua, usando US como método para guiar la implantación de dicho catéter.

\section{DESCRIPCIÓN DEL CASO}

Paciente de sexo femenino, 4 años de edad y $16 \mathrm{~kg}$ de peso, con diagnóstico de rabdomiosarcoma en muslo izquierdo y metástasis ganglionar inguinal ipsilateral, fue programada de forma electiva para resección de recidiva tumoral local en tejidos blandos, vaciamiento ganglionar inguinal y colocación de catéteres para braquiterapia. La paciente había recibido quimioterapia en los meses previos, no tenía antecedentes de alergia a medicamentos y en la intervención previa de su tumor no tuvo complicaciones anestésicas. Sus exámenes prequirúrgicos mostraban hemoglobina $10,9 \mathrm{~g} / \mathrm{dl}$, hematocrito $31,7 \%$, conteo plaquetario $213.000 / \mu 1$, TPT 24,5 segundos, TP 11,3 segundos e INR 1,06. Se reservó 1 unidad de glóbulos rojos empaquetados en su evaluación preanestésica. Se discutió

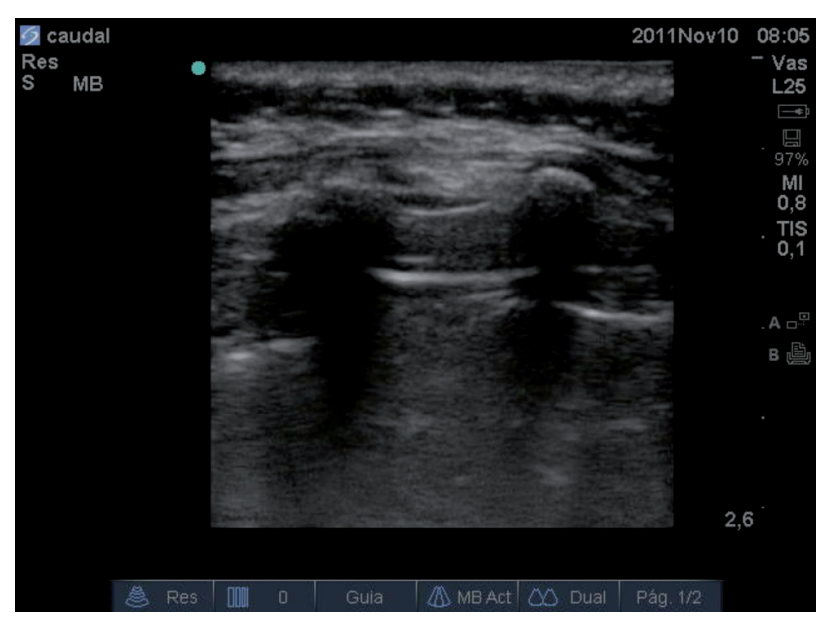

Fig. 1.

con su madre en la evaluación preanestésica la posibilidad de ofrecer una técnica regional continua para el manejo del perioperatorio, destacando su papel en el control analgésico post-quirúrgico. El consentimiento informado fue diligenciado. La paciente tenía una vena periférica canalizada a su ingreso al quirófano y se realizó una inducción anestésica convencional con fentanil $2 \mu \mathrm{g} / \mathrm{kg}$, lidocaína $1 \mathrm{mg} / \mathrm{kg}$, propofol $2 \mathrm{mg} / \mathrm{kg}$, dexametasona $0,15 \mathrm{mg} / \mathrm{kg}$ y cisatracurio $2 \mathrm{mg} / \mathrm{kg}$, intubación orotraqueal con tubo 5.0 sin balón, mantenimiento anestésico balanceado con remifentanilo en infusión titulada y sevoflurano a 0,8 MAC, ventilación mecánica con volumen corriente de $7 \mathrm{ml} / \mathrm{kg}$, frecuencia respiratoria 22 por minuto, PEEP $5 \mathrm{cmH}_{2} \mathrm{O}$ y $\mathrm{FiO}_{2} 70 \%$. Después la paciente se posicionó en decúbito lateral izquierdo y flexión de las caderas. En esta posición realizamos un escaneo previo de la zona caudal utilizando un equipo SonoSite M-Turbo ${ }^{\circledR}$ con transductor lineal L25x de 13-6 MHz, donde se identificaron los distintas estructuras a ese nivel incluida la membrana sacro-coxígea y los cuernos sacros (Fig. 1). Posteriormente se realizó antisepsia con clorhexidina al $4 \%$ en zona lumbosacra y perineal y se cubrió con campos estériles. Con técnica aséptica nuevamente se escanearon las estructuras y con el transductor en el eje longitudinal al sacro y se procedió a abordar el espacio caudal en plano con una aguja $20 \mathrm{G}$ de $5 \mathrm{~cm}$ para técnica continua (Set StimuLong Sono catheter PAJUNK®) (Fig. 2). Una vez allí y bajo visión ultrasonográfica directa se pasó el catéter a través de la aguja, avanzándolo hasta una distancia de $8 \mathrm{~cm}$, quedando $3 \mathrm{~cm}$ en el espacio caudal, se retiró la aguja y se instaló el filtro del equipo. Visualizando la punta del catéter en el espacio caudal y con el modo Doppler color activado en el quipo se inyectó una dosis bolo de $16 \mathrm{ml}$ de mezcla anestésica (bupivacaína $0,5 \%$ con epinefrina 1:200.000, $8 \mathrm{ml}+$ solución salina $0,9 \%$ estéril 8 $\mathrm{ml}$ ) visualizando la estela de color (efecto Doppler) confirmando flujo a nivel del espacio epidural (Fig. 3). Finalmen- 


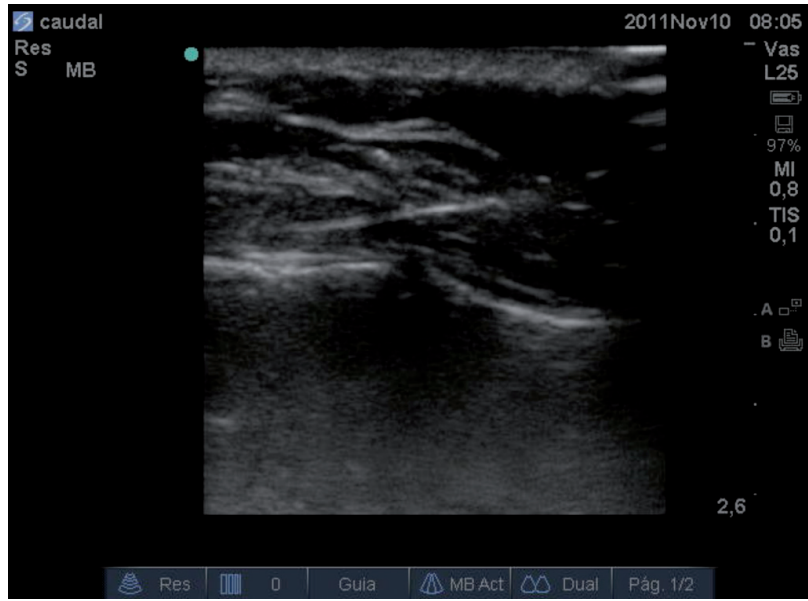

Fig. 2.

te la porción externa del catéter fue dirigida hacia cefálico y se cubrió con apósitos estériles 3M $\mathrm{M}^{\mathrm{TM}}$ Tegaderm ${ }^{\mathrm{TM}}$ (Fig.4).

Después de la colocación del catéter, la paciente se reposicionó en decúbito supino y fue llevada a cabo la cirugía donde se encuentra conglomerado tumoral sobre vasos inguinales los cuales se liberaron, se envió un espécimen inicial reseccionado para biopsia por congelación, que resultó positiva en los márgenes y se decidió ampliar dicha resección con nuevo resultado negativo. Se resecó vasto medial y crural completamente en la zona afectada. Se dejaron 8 catéteres para braquiterapia. La pérdida sanguínea fue aproximadamente de $50 \mathrm{ml}$ y no fue transfundida. La duración de la cirugía fue de 4 horas y 15 minutos y los requerimientos anestésicos durante su realización fueron mínimos. Al finalizar la cirugía se aplicaron $2 \mathrm{mg}$ de morfina intravenosos, se extubó sin complicaciones y fue llevada a recuperación para vigilancia donde se inició la infusión por el catéter caudal a $3 \mathrm{ml} / \mathrm{h}$ de una mezcla de fentanilo

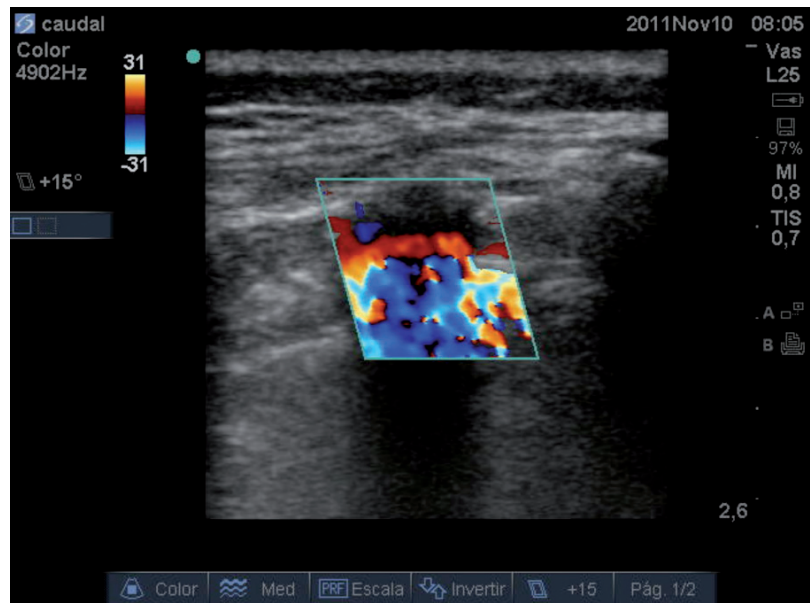

Fig. 3.

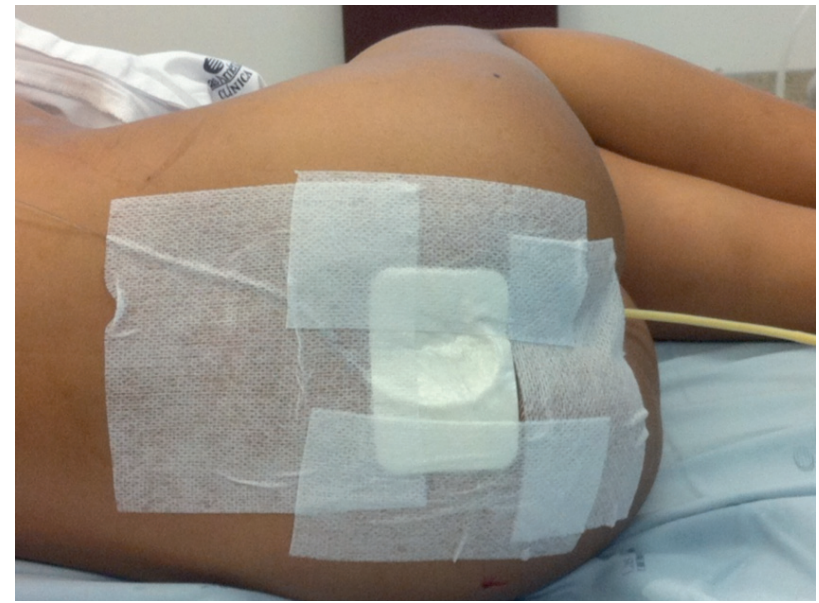

Fig. 4.

$3 \mu \mathrm{g} / \mathrm{ml}$ y bupivacaína al $0,1 \%$ sin epinefrina. La paciente permaneció estable en recuperación durante 3 horas más sin requerir suplementos analgésicos y luego fue trasladada a la habitación de hospitalización general pediátrica. Los medicamentos analgésicos que recibió adicionalmente durante su hospitalización fueron acetaminofeno $240 \mathrm{mg}$ cada 6 horas vía oral y diclofenaco $15 \mathrm{mg}$ cada 12 horas vía intravenosa por horario, con rescates de hidromorfona a necesidad. El seguimiento analgésico hasta el alta se detalla en la tabla I. No fueron requeridos rescates con opioides. El catéter fue retirado al tercer día postoperatorio después de 70 horas de colocado sin complicaciones. El mismo día se dio de alta a la paciente con adecuado control del dolor, medicación oral e instrucciones.

\section{DISCUSIÓN}

El caso presentado ilustra la implantación de un catéter caudal para técnica regional continua en una paciente con rabdiomioscarcoma y metástasis ganglionar inguinal en la cual se realizó extracción de lesión tumoral en muslo, implantación de agujas para braquiterapia y vaciamiento inguinal. La decisión de implantar un catéter caudal continuo fue motivada por el hecho de la permanencia de los catéteres para braquiterapia, lo cual suponía un estímulo adicional que no sería cubierto por una dosis única; además la presencia de conglomerados ganglionares inguinales hacían poco atractiva la opción de un bloqueo femoral dosis única o continua. El bloqueo caudal es la técnica regional más popular en niños, sobre todo la inyección única, por su alta tasa de éxito, fácil aprendizaje y baja incidencia de complicaciones severas (7-9). La técnica pediátrica básica por referencias anatómicas que describen los libros clásicos consiste en colocar el paciente en una posición decúbito lateral o prono con un rollo bajo sus crestas ilíacas, se pal- 
TABLA I. SEGUIMIENTO ANALGÉSICO

\begin{tabular}{cccccc}
\hline Día & Hora & Escala de dolor & Rata de infusión & Escala de sedación & Efectos secundarios \\
\hline Cirugía & $16: 00$ & 0 & $3 \mathrm{ml} / \mathrm{h}$ & Despierto-tranquilo & Ninguno \\
$1^{\circ}$ POP & $08: 00$ & 0 & $3 \mathrm{ml} / \mathrm{h}$ & Despierto-tranquilo & Parestesias en MID \\
$1^{\circ}$ POP & $16: 00$ & 0 & $2 \mathrm{ml} / \mathrm{h}$ & Despierto-tranquilo & Ninguno \\
$1^{\circ} \mathrm{POP}$ & $22: 00$ & 1 & $2 \mathrm{ml} / \mathrm{h}$ & Despierto-tranquilo & Ninguno \\
$2^{\circ} \mathrm{POP}$ & $06: 00$ & 1 & $2 \mathrm{ml} / \mathrm{h}$ & Despierto-tranquilo & Ninguno \\
$2^{\circ}$ POP & $18: 00$ & 10 & $2 \mathrm{ml} / \mathrm{h}$ & Despierto-tenso & Ninguno \\
$2^{\circ}$ POP & $22: 00$ & 0 & $2 \mathrm{ml} / \mathrm{h}$ & Despierto-tranquilo & Ninguno \\
\hline
\end{tabular}

pan los cuernos sacros con un separación de $0,5-1 \mathrm{~cm}$ al mover los dedos en dirección medial a lateral. Cuando los cuernos sacros no son palpados fácilmente o no son prominentes, podría facilitarse palpar el espacio intervertebral L5-S1 en la línea media y entonces palpar en una dirección caudal hasta que el hiato sacro es alcanzado. Una aguja de bisel corto No. $22 \mathrm{G}$ se dirige cefálicamente en un ángulo de $45-75^{\circ}$ con la piel hasta sentir un "pop" atravesando el ligamento sacrocoxígeo accediendo al canal caudal, el cual es continuo con el espacio epidural. Si se encuentra hueso antes del ligamento sacrocoxígeo la aguja debe ser retirada varios milímetros, cambiar el ángulo de la aguja aproximadamente a $30^{\circ}$ y nuevamente avanzada cefálicamente hasta perforar el ligamento sacrocoxígeo. Cuando la aguja es avanzada un poco más, el hueso es contactado y debe ser nivelada al plano paralelo a la espalda del niño antes del avance. Una vez la aguja está en el espacio caudal es avanzada unos poco milímetros, por el riesgo de puncionar el saco dural. Con la aspiración negativa para sangre o LCR se aplica una dosis de prueba de anestésico local con atención a cambios hemodinámicos y en el ECG, si estos no se presentan se termina de inyectar lentamente el resto del anestésico sin resistencia y advirtiendo cualquier edema subcutáneo adyacente (1). Sin embargo, la técnica clásica, por referencias anatómicas, tiene algunas limitaciones que potencialmente podrían, aunque raramente, llevar a complicaciones y que pueden ser superadas con el uso de US, tales como inyección intratecal, intravascular, intraósea, subcutánea y rectal del AL $(10,11)$, contacto con el hueso y falla de la técnica por aplicación del AL fuera del espacio caudal (4). En la técnica clásica, la capacidad para localizar el hiato sacro y definir las variantes anatómicas es el principal factor que determina la seguridad y el éxito de la técnica. Dentro de las ventajas que brinda la US en este procedimiento se cuenta que brinda información de la anatomía sacra subyacente donde abundan las variantes anatómicas (12) y donde muchas veces según la edad del paciente es difícil la palpación de las estructuras; permite la ubicación del sitio de punción, hay mejoría en la tasa de éxito en el primer intento y disminuye el número de punciones (13); además permite la visualización directa de la aguja o catéter para guiar su colocación en tiempo real y la diseminación del AL en el espacio caudal-epidural $(4,14-$ 16). Una ventaja adicional importante que ofrece la US es poder visualizar el desplazamiento anterior de la dura madre, la turbulencia y dilatación del espacio caudal a la inyección, y mediante el modo Doppler color el momento de la aplicación del anestésico en tiempo real que confirma el sitio donde se está aplicando $(4,17)$, como se visualiza en la fig. 2. La estela de color que se genera a la aplicación es un signo que disminuye la probabilidad a cerca de 5-9\% de que el inyectado sea intravascular (2), lo cual es muy importante sobre todo en un paciente anestesiado (como se realiza comúnmente en niños) debido al enmascaramiento de los signos iniciales de toxicidad. En niños de edad similar a nuestra paciente, se ha descrito que la US tiene una sensibilidad del 96,3 vs. $57,5 \%$ y un valor predictivo negativo del 40 vs. $5 \%$ comparado con el "swoosh test" para confirmar el sitio adecuado de aplicación, siendo incluso dentro de los parámetros ecográficos la turbulencia más sensible que el flujo Doppler color (95 vs. 78,8\%) (18).

Las complicaciones descritas asociadas a la analgesia caudal continua son raras y generalmente no son graves, entre las más reconocidas están el síndrome compartimental, el cual ha sido detectado como dolor a pesar de una infusión previa normofuncionante (19), bloqueo motor con zonas de presión o neuropraxias, lo cual se puede evitar posicionando adecuadamente, acolchonando y movilizando regularmente las extremidades, la toxicidad por $\mathrm{AL}$ por acumulación en el tiempo, por lo que se deben evitar infusiones que excedan los $0,3-0,4 \mathrm{mg} / \mathrm{kg} / \mathrm{h}$ especialmente después de 24 horas. Los efectos asociados a los opioides agregados a la infusión, como depresión respiratoria, prurito y náuseas son otros eventos indeseables además de retención urinaria que ocurre hasta en el 30\% de pacientes, todos son dependientes de la dosis y se debe usar la mínima efectiva (14). A pesar de que hasta el $35 \%$ de los catéteres caudales no tunelizados se colonizan, las infecciones clí- 
Rev. Soc. Esp. del Dolor, Vol. 20, N. ${ }^{\circ}$ 1, Enero-Febrero 2013

nicas epidurales son raras $(20,21)$. La infección local fue la complicación más frecuentemente encontrada con las infusiones epidurales analgésicas por la auditoria nacional epidural pediátrica realizada en el Reino Unido e Irlanda, sin embargo se vio que los catéteres caudales no fueron asociados con incremento en la incidencia de infecciones comparado con los lumbares y dorsales, pero esto no fue estadísticamente significativo. El germen más implicado fue Staphylococcus aureus, los catéteres permanecieron en promedio 77 horas y varias infecciones junto con un absceso se detectaron después de un día de retirado el catéter (19). Para la realización del procedimiento, se ha sugerido el uso de precauciones de barrera máximas, como uso de gorro, bata, mascarilla, guantes y campos estériles (22), el uso de clorhexidina al $0,5 \%$ en alcohol para la preparación de la piel, así como tunelización del catéter para disminuir el riesgo de colonización e infección $(23,24)$, aunque esto último requiere más evidencia. Nosotros utilizamos todas las medidas asépticas para el procedimiento, preparamos la piel con clorhexidina a una mayor concentración, retiramos el catéter antes de 72 horas, y a pesar de no haberlo tunelizado, no tuvimos ninguna complicación seria con esta paciente en los días posteriores.

Uno de los objetivos de reportar este caso describiendo algunas de las bondades que tiene la US sobre la técnica clásica por referencias anatómicas, es incentivar su uso cada vez más en nuestro medio, mejorar la curva de entrenamiento en esta técnica más segura y sobre todo usarlo siempre que se tenga el recurso en casos donde la técnica clásica esté limitada por distorsión anatómica o palpación difícil del hiato sacro, sin dejar de beneficiar los pacientes de esta excelente medida para manejo del dolor intra y postoperatorio. Schwartz D y cols. pudieron beneficiar a un paciente de esta técnica gracias a que la US les permitió superar la distorsión anatómica por edema de la zona caudal por intentos fallidos previos con la técnica clásica (17), y aunque nosotros en esta paciente no tuvimos esta situación, se presta para ilustrar la técnica e iniciar una curva de aprendizaje que permita abordar de forma más segura otros casos subsiguientes más retantes que no son infrecuentes. Se exhorta a las instituciones de nuestro medio para adquirir equipos de US e implementar estrategias de entrenamiento a los anestesiólogos y así fomentar la anestesia regional como un pilar en la dinámica del programa quirúrgico lo cual mejora la calidad y seguridad en la atención a un coste-beneficio favorable (25).

\section{CONCLUSIÓN}

El uso de US para la implantación de catéteres epidurales caudales y para técnicas de una sola inyección, es una excelente alternativa a las técnicas tradicionales a ciegas, brindando ciertas ventajas sobre las mismas. Por tanto el empleo de US debe ser una herramienta cada vez más disponible para el anestesiólogo para permitir el empleo de esta y otras técnicas regionales mejorando la calidad en la atención.

\section{CORRESPONDENCIA:}

Carlos Eduardo Restrepo-Garces

Director Unidad de Alivio del Dolor

Departamento de Anestesiología

Clínica Las Américas. Instituto de Cancerología.

Torre Médica Las Américas, Cr 80 2ª 80-140.

Medellín, Colombia

e-mail: carlosedo75@gmail.com

\section{BIBLIOGRAFÍA}

1. Polaner DM, Suresh S, Coté CJ. Regional Anesthesia. In: Coté CJ, Lerman J, Todres ID (editors). A practice of anesthesia for infants and children. New York: Saunders Elsevier; 2009. p. 867-910.

2. Najman IE, Frederico TN, Segurado AV, et al. Caudal epidural anesthesia: an anesthetic technique exclusive for pediatric use? Is it possible to use it in adults? What is the role of the ultrasound in this context? Rev Bras Anestesiol. 2011 Jan-Feb;61(1):95-109.

3. Menzies R, Congreve K, Herodes V, et al. A survey of pediatric caudal extradural anesthesia practice. Paediatr Anaesth. 2009 Sep;19(9):829-36.

4. Tsui BC, Suresh S. Ultrasound imaging for regional anesthesia in infants, children, and adolescents: a review of current literature and its application in the practice of neuraxial blocks. Anesthesiology. 2010 Mar;112(3):719-28. Review. Lönnqvist PA. Is ultrasound guidance mandatory when performing paediatric regional anaesthesia? Curr Opin Anaesthesiol. 2010 Jun;23(3):337-41. Review.

5. Tsui BC, Pillay JJ. Evidence-based medicine: Assessment of ultrasound imaging for regional anesthesia in infants, children, and adolescents. Reg Anesth Pain Med. 2010 MarApr;35(2 Suppl):S47-54. Review.

6. Dalens B, Hasnaoui A. Caudal anesthesia in pediatric surgery: success rate and adverse effects in 750 consecutive patients. Anesth Analg 1989;68:83-9.

7. Giaufre E, Dalens B, Gombert A. Epidemiology and morbidity of regional anesthesia in children: a one-year prospective survey of the French-Language Society of Pediatric Anesthesiologists. Anesth Analg 1996;83:904-12.

8. Schuepfer G, Konrad C, Schmeck J, et al. Generating a learning curve for pediatric caudal epidural blocks: an empirical evaluation of technical skills in novice and experienced anesthetists. Reg Anesth Pain Med 2000;25:385-8.

9. Giaufre E. Risks and complications of regional anaesthesia in children. Best Prac Res Anaesthesiol 2000;14:659-71.

10. Suresh S, Wheeler M. Practical pediatric regional anesthesia. Anesthesiol Clin North Am 2002;20:83-113.

11. Ellis H, Feldman S, Harrop-Griffiths W. The vertebrae and sacrum. In: Ellis H, Feldman S, Harrop-Griffiths W (editors). Anatomy for Anaesthetists. Massachusetts, USA: Blackwell Science Ltd; 2004. p. 97-118.

12. Manoj K. Karmakar. Ultrasound for central neuraxial blocks. Tech Reg Anesth Pain Manag, 2009;13:161-70. 
13. Polaner DM, Drescher J. Pediatric regional anesthesia: what is the current safety record? Paediatr Anaesth. 2011 Jul;21(7):737-42.

14. Bauer M, George JE 3rd, Seif J, Farag E. Recent advances in epidural analgesia. Anesthesiol Res Pract. 2012; 2012: 309219. Epub 2011 Nov 24.

15. Rubin K, Sullivan D, Sadhasivam S. Are peripheral and neuraxial blocks with ultrasound guidance more effective and safe in children? Paediatr Anaesth. 2009 Feb;19(2):92-6. Review.

16. Schwartz D, Raghunathan K, Dunn S, et al. Ultrasonography and pediatric caudals. Anesth Analg. 2008 Jan; 106(1):97-9.

17. Raghunathan K, Schwartz D, Connelly NR. Determining the accuracy of caudal needle placement in children: a comparison of the swoosh test and ultrasonography. Paediatr Anaesth. $2008 \mathrm{Jul} ; 18(7): 606-12$.

18. Llewellyn N, Moriarty A. The national pediatric epidural audit. Pediatr Anesth 2007;17:520-33.
19. Kost-Byerly S, Tobin JR, Greenberg RS, et al. Bacterial colonization and infection rate of continuous epidural catheters in children. Anesth Analg. 1998 Apr;86(4):712-6.

20. Strafford MA, Wilder RT, Berde CB. The risk of infection from epidural analgesia in children: a review of 1620 cases. Anesth Analg. 1995 Feb;80(2):234-8.

21. Association of Anaesthetists of Great Britain and Ireland. Infection control in anaesthesia. Anaesthesia. 2008 Sep;63 (9):1027-36.

22. Kinirons B, Mimoz O, Lafendi L, et al. Chlorhexidine versus povidone iodine in preventing colonization of continuous epidural catheters in children: a randomized, controlled trial. Anesthesiology. 2001 Feb;94(2):239-44.

23. Fujinaka W, Hinomoto N, Saeki S, et al. Decreased risk of catheter infection in infants and children using subcutaneous tunneling for continuous caudal anesthesia. Acta Med Okayama. 2001 Oct;55(5):283-7.

24. Griffin J, Nicholls B. Ultrasound in regional anaesthesia. Anaesthesia. 2010 Apr;65(Suppl 1):1-12. 